\title{
Role of Recent Imaging Modalities in Diagnosis of Diabetic Foot
}

\author{
Mohamed Kamal Hamed Elsharkawy, Mokhtar Ragab Ramadan, Mostafa Mahrose Abd Elhamed \\ Department of Radiodiagnosis, Faculty of Medicine, Alazhar University \\ Corresponding author: Mostafa Mahrose Abd Elhamed, Mobile: 002001023136936, E-Mail: sheeekooo2030@ gmail.com
}

\begin{abstract}
Background: osteomyelitis and septic arthritis complicate up to one third of diabetic foot infections that require hospitalization and often result from contaminated soft tissues. Early diagnosis is difficult to achieve through noninvasive imaging studies. Nuclear medicine and radiologic imaging techniques, although commonly used, may lack accuracy. CT is used routinely because it is widely available, but it is of limited diagnostic value in early stages of acute osteomyelitis of the foot and in patients with diabetic osteoarthropathy. MRI, because of its high sensitivity and specificity, is considered the modality of choice for diagnosing osteomyelitis of the foot and for identifying associated soft-tissue abnormalities such as cellulitis, phlegmon, abscess, sinus tracts and ulcers. Aim of the work: this study aimed to assess the role of recent imaging modalities such as high resolution ultrasound, CTA, MRI, MRA and PET/CT in the early detection and /or improving the prognosis of diabetic foot.

Conclusion: the major complication facing patients with diabetic foot is amputation that is why tight glycemic control is mandatory. In addition to routine screening for early detection of diabetic foot complications can be helpful. With advancement of radiological techniques, the prognosis of diabetic foot has improved.
\end{abstract}

Keywords- CTA, MRI, MRA, PET/CT, diabetic foot

\section{Introduction}

Diabetes Mellitus is a complex metabolic disorder characterized by hyperglycemia, micro vascular disease and peripheral sensory neuropathy rendering skin susceptible to injury. Furthermore, it blunts healing and predisposes the extremity to increase occurrence of infections ${ }^{(1)}$. Associated complications in the lower limb include Charcot's joint (progressive destructive arthropathy caused by neuropathy), ulceration, infection, gangrene and amputation (2). Clinical assessment of the swollen, red and hot foot for the presence of osteomyelitis is poor, even in the presence of softtissue ulcer. Therefore, the radiologist is often asked to participate in the evaluation (3). Assessment of the lower limb vessels to detect the degree of vascular affection is crucial for determining the prognosis and surgical decision of any intervention. The vessels of the lower limbs can be examined in order to identify segments of stenosis, or occlusion with Doppler ultrasound, conventional angiography, CT, MR angiography (4).

The foot is mostly superficial structures and thus is particularly suitable for high-resolution ultrasound evaluation. Direct patient contact allows accurate identification of the site of symptoms and allows a pertinent history to be obtained. Moreover, Ultrasound offers real-time comparison with the contra-lateral side ${ }^{(5)}$. By the Doppler without resorting to intravenous contrast injection we can be assessed The internal vascularity of the pathology - e.g., synovitis. Magnetic Resonance Imaging (MRI) also has many inherent advantages such as the imaging of deeper soft tissue structures, bones, and joints, and it is fewer operators dependent. However, MRI and ultrasonography are, complementary imaging techniques and the choice of modality will often depend upon local expertise, accessibility and cost ${ }^{(5)}$. In comparison with imaging modalities such as CT, MRI and ultrasound, FDG-PET is advantageous because it offers full body coverage, high sensitivity, an absence of artifacts from metallic hardware. Fluorine-18 (F18) Fluoro-Deoxy Glucose-Positron Emission Tomography (FDG-PET) represents a promising imaging technique and has shown many advantages compared to conventional radionuclide imaging methods. FDG-PET has the highest diagnostic accuracy for confirming or excluding chronic osteomyelitis ${ }^{(6)}$.

\section{Aim of the work}

This study aimed to assess the role of recent imaging modalities such as high resolution ultrasound, CTA, MRI, MRA and PET/CT in the 
early detection and /or improving the prognosis of diabetic foot.

Anatomy of the Foot

Bones of the foot (Fig.1):

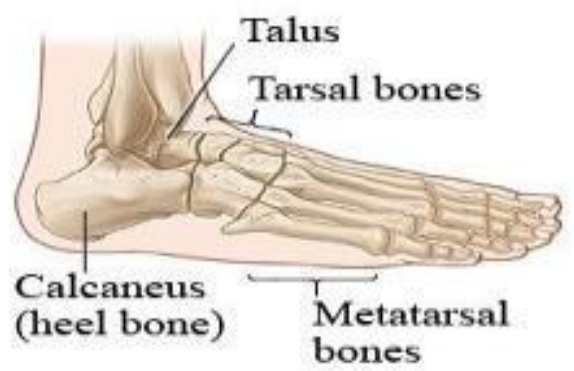

o Healthwice, incorporated

Fig. 1: bones of the ankle and foot ${ }^{(7)}$

Joints of the foot:

1. The ankle joint.

2. Inter tarsal joints.

3. Tarso- Inter-metatarsal joints.

4. Metatarso-phalangeal Articulations.

5. Inter-phalangeal joints ${ }^{(8)}$.

Muscles and tendons of the foot (Fig. 2):

The tendons about the ankle are divided into anterior, posterolateral, postero-medial and posterior groups.

a) The anterior (extensor) group: is contained within the space bounded by the deep fascia anteriorly and posteriorly by the
The foot is composed of seven tarsal bones that are arranged into proximal (talus and calcaneus), intermediate (navicular) and distal groups (cuboid and three cuneiforms).The foot includes five metatarsals and five digits and their phalanges ${ }^{(7)}$. (a)

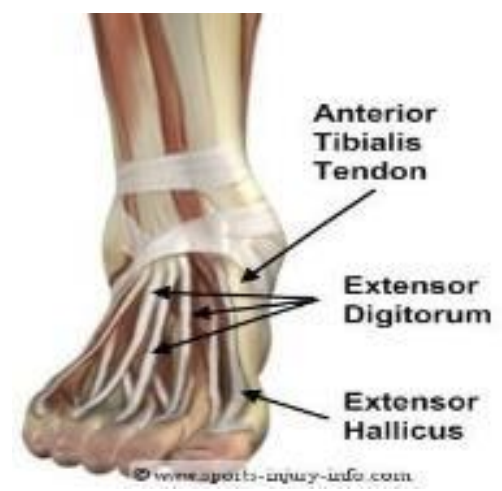

interosseous membrane. From medial to lateral lie the tibialis anterior, extensor hallucis longus, and extensor digitorum longus and peroneus tertius.

b) The postero-medial group of tendons lies at the level of the medial malleolus. From anteromedial to postero-medial, they are the tibialis posterior, flexor digitorum longus, and flexor hallucis longus. They are enclosed within their own synovial sheath deep to the flexor retinaculum.

c) The postero-lateral group is composed of the peroneus longus and brevis.

d) The posterior group is composed of the Achillis tendon ${ }^{(5)}$.

(b)

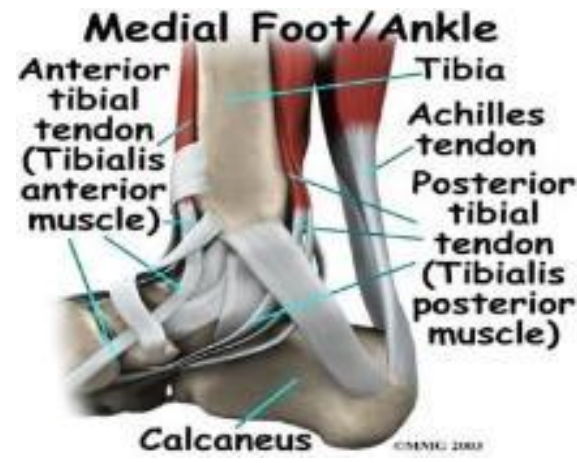




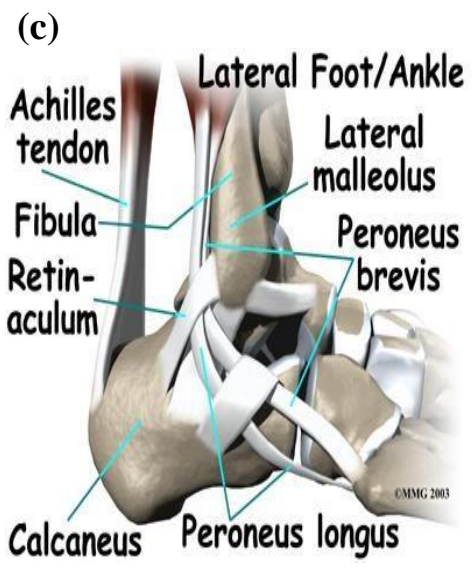

Fig. 2: the muscles, tendons and retinaculae around the ankle joint: (a) anterior, (b) medial, (c) lateral, and (d) posterior views ${ }^{(\mathbf{5})}$

The sole of the foot has extremely thick subcutaneous tissue with fibrous bands extending and anchoring it to the plantar aponeurosis which is composed of dense bands arranged largely along the long axis of the foot ${ }^{(5)}$.

The muscles along the sole of the foot are divided into four distinct layers (Fig.3).

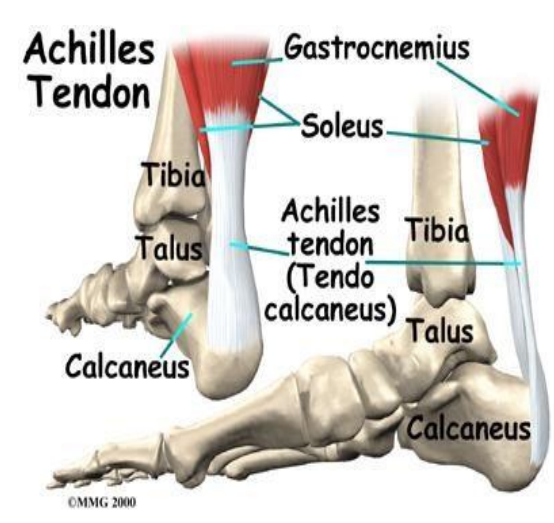

1. The first layer is just deep to the plantar aponeurosis.

2. The second layer includes the long flexor tendons..

3. The third layer is composed of three short muscles.

4. The fourth layer includes the interossei seen within the intermetatarsal spaces, as well as, the tendons of the tibialis posterior and peroneus longus ${ }^{(5)}$. (a)

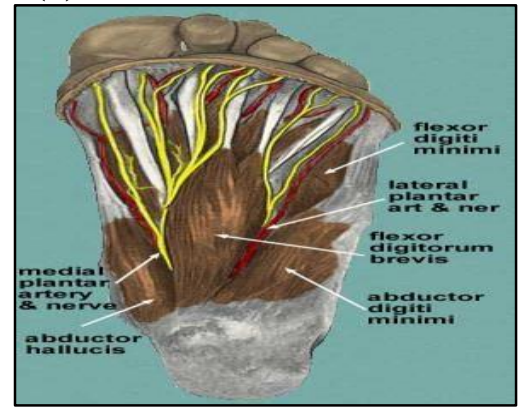

(c)

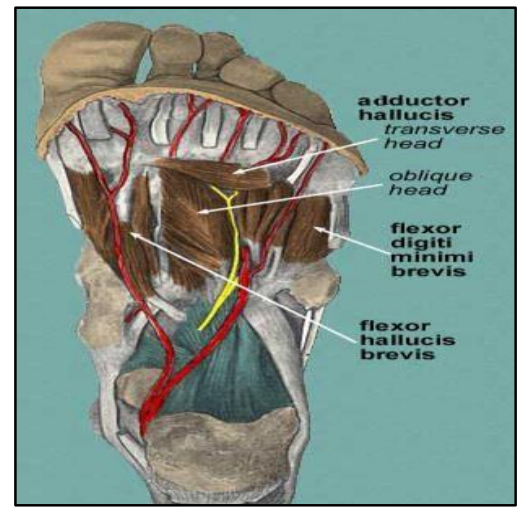

(b)

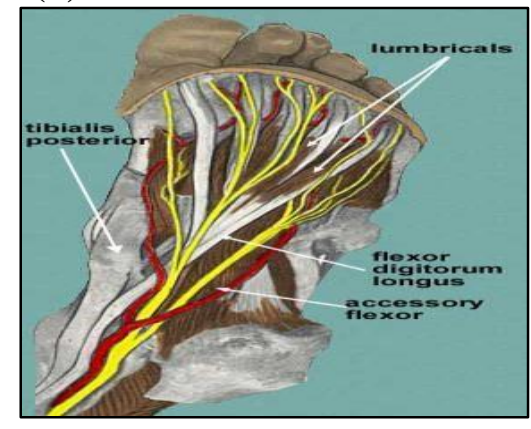

(d)

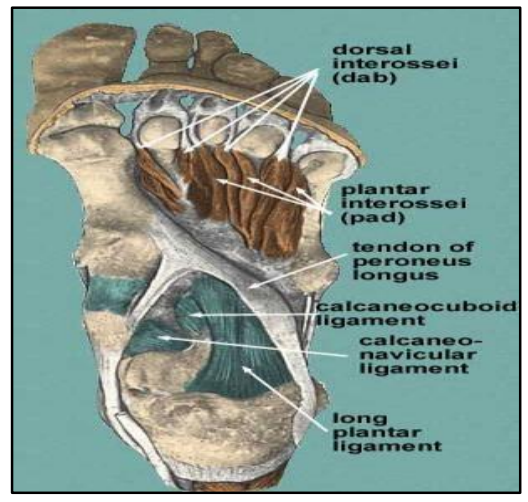

Fig. 3: muscle layers of the sole (a) $1^{\text {st }},($ b $) 2^{\text {nd }},(c) 3^{\text {rd }},(d) 4^{\text {th }}$ layer ${ }^{(5)}$

Normal imaging anatomy of the foot:

1) X-Ray: 
On Antero-Posterior (AP) radiographs, of the ankle joint the tibio-talar surfaces are seen to be parallel to each other and perpendicular to the shaft of the tibia. The articular surfaces of the malleoli on each side are symmetrical about a line through the shaft of the tibia, each making an equal angle with the inferior surface of the tibia. The medial and lateral ligaments of the ankle joint are not visible on plain radiographs but their integrity can be deduced from normal joint alignment in standard views and, where appropriate, stress views. Where the distance between the medial malleolus and the talus is increased then disruption of the deltoid ligament can be diagnosed, and where there is increased distance between the lateral malleolus and the talus without bony injury this is due to disruption of the lateral ligaments. In general loss of parallelism of the articular surface is significant. The term tri-malleolar fracture is sometimes used, although there are only two malleoli. The third 'malleolus' is the posterior lip of the tibial articular surface ${ }^{(9)}$.

\section{2) Computerized Tomography:}

This can be combined with arthrography to assess the bony components of the ankle joint. Direct coronal and axial images are routinely acquired in slices $2 \mathrm{~mm}$ thick ${ }^{(9)}$.

\section{Normal MRI of the foot:}

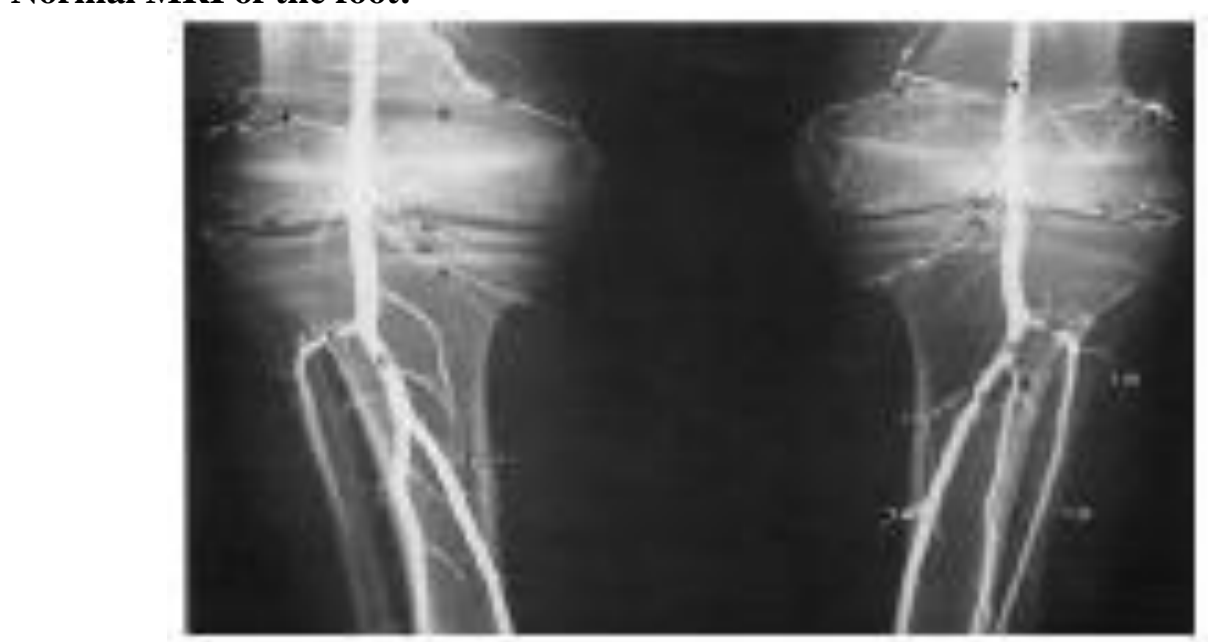

Fig .4: normal infra popliteal angiography ${ }^{(9)}$
The normal signal intensity of the different tissues of the ankle and foot depends on the pulse sequence. Tendons, ligaments, and cortical bone are hypo-intense in practically any pulse sequence. Muscles and nerves are seen as intermediate signal intensity structures on all pulse sequences. On spin-echo images, arteries demonstrate hypo-intensity due to flow void and veins show hyper-intensity due to slow flow. On gradient-echo images, both arteries and veins are hyper-intense. Depending on the percentage of fat, hematopoietic marrow, and trabecular bone, the bone marrow has signal intensity similar to that of the subcutaneous fat. In young individuals, islands of hematopoietic marrow can be present in the distal tibia. Hematopoietic red marrow is relatively hypo-intense on T1- and T2-weighted spin-echo pulse sequences and is hyper-intense on STIR images and T2 fat-saturated sequences ${ }^{(\mathbf{1 0})}$.

Angiography appearance of the arteries of the foot:

The three main vessels of the lower leg lie over and between the shafts of the tibia and fibula.

Slight internal rotation of the foot helps to project the tibia away from the fibula and allows the vessels to be seen between them. The dorsalis pedis artery is best seen on lateral views of the foot and the medial and lateral plantar arteries and the plantar arch are seen on dorsi-plantar views of the foot (Figs. 4, 5) ${ }^{(9)}$. 
1. Popliteal artery

3. Medial superior genicular artery

5. Middle genicular artery

7. Lateral inferior genicular artery

9. Tibioperoneal trun
2. Descending genicular artery

4. Lateral superior genicular artery

6. Medial inferior genicular artery

8. Anterior tibial artery

10. Posterior tibial artery

\section{Peroneal artery}

12. Muscular branches ${ }^{(9)}$.

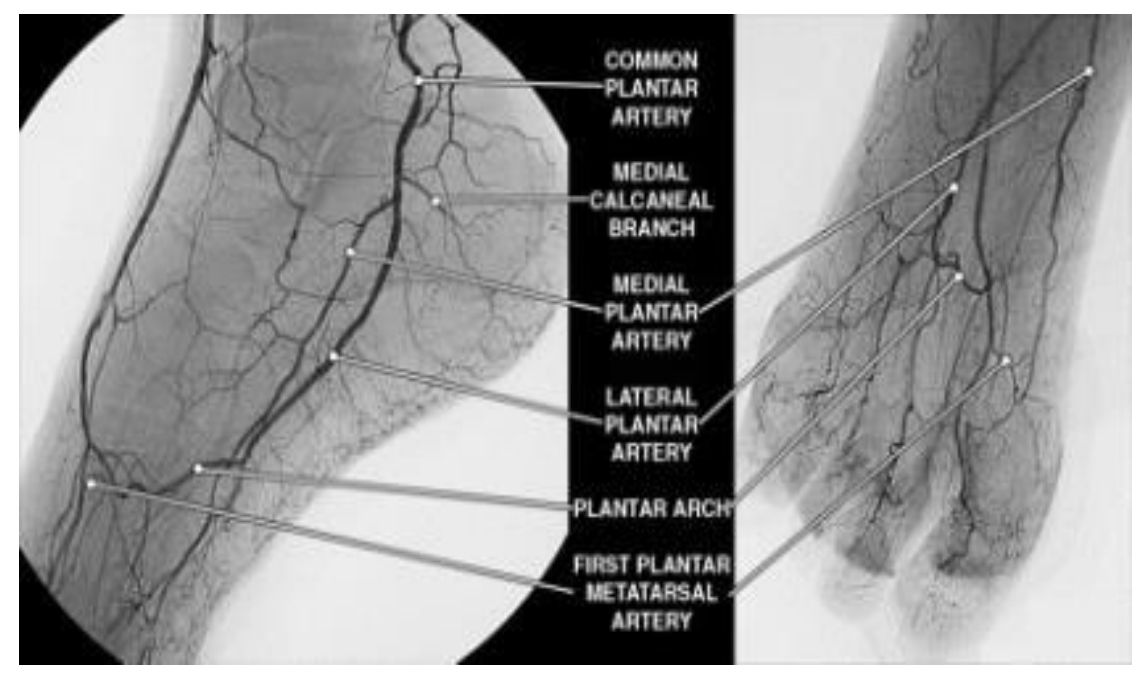

Fig 5: posterior pedal circulation. The major posterior pedal arteries are depicted in lateral oblique (left) and antero-posterior (right) angiographic projections ${ }^{\mathbf{( 1 0 )}}$

\section{Path physiology of the diabetic foot}

Diabetic foot is defined as the presence of infection, ulceration and/or destruction of deep tissues associated with neurologic abnormalities and various degrees of peripheral arterial disease in the lower limb in patients with diabetes ${ }^{(11)}$. Trauma and loss of protective sensation or peripheral arterial disease are the major contributors to foot ulceration, and diabetic neuropathy is the common denominator in almost 90\% of diabetic foot ulcers ${ }^{(11)}$.

\section{Role of Glycemic Control in Preventing}

\section{Complications:}

A paradigm shift in diabetes treatment occurred in 1993 with publication of the results of the Diabetes Control and Complications Trial (DCCT), the first major trial to examine the effects of attempted glucose normalization (tight or intensive diabetic control) on the incidence of complications. In this study of individuals with Type $1 \mathrm{DM}$, intensive treatment reduced micro-vascular complications (retinopathy, nephropathy, neuropathy) by $60 \%$. A subsequent study in Type 2 DM demonstrated a $25 \%$ decrease in micro-vascular complications (retinopathy, nephropathy) with improved glycemic control ${ }^{(\mathbf{1 2})}$. While, the importance of glycemic control in influencing the occurrence of micro-vascular complications is uncontroversial, genetic factors also clearly play a role. For example, evidence from a variety of studies suggests that approximately $40 \%$ of type 1 diabetics may be particularly susceptible to the development of severe micro-vascular complications ${ }^{(\mathbf{1 2})}$. 


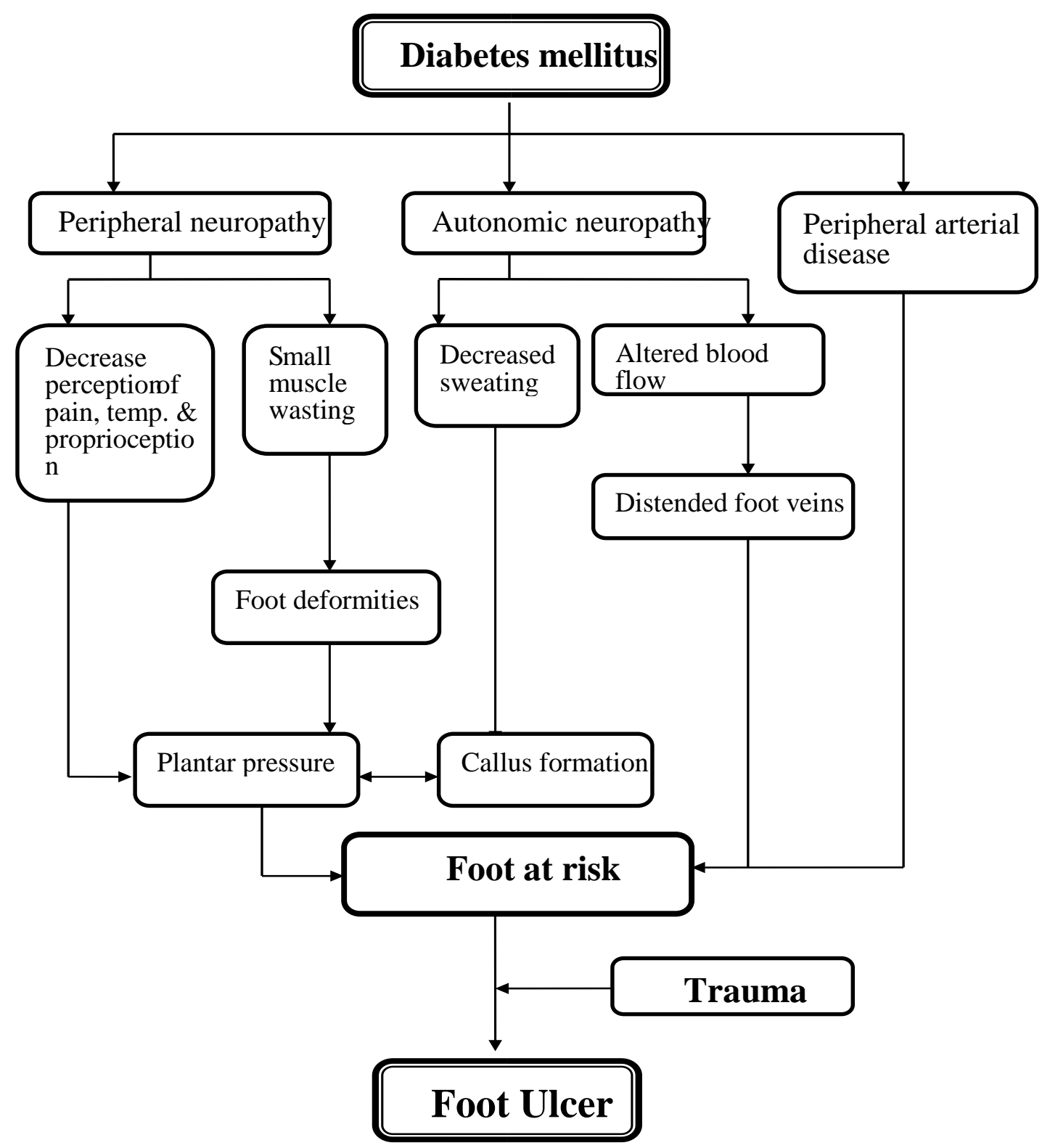

Fig. 6: the pathways to foot ulceration ${ }^{(11)}$

Path-physiology of peripheral arterial disease (PAD):

Foot blood flow supplies three major tissue components: skin, muscle, and bone. On the other hand, muscle flow is related to the contractile activity, rising strikingly in parallel with oxygen consumption during intrinsic foot muscle contraction. Skin blood flow is determined principally by body core and environmental temperature, rising with the need to dissipate heat (5). In people with diabetes, the risk of PAD increases by age, duration of diabetes, and presence of peripheral neuropathy. It is important to note that diabetes is most strongly associated with femoral, popliteal and tibial PAD ${ }^{(13)}$. Platelet function is abnormal in diabetes as well. Expression of both glycoprotein Ib and IIb/IIIa is increased, augmenting both platelet- von Willebrand factor and platelet-fibrin interaction. Hyperglycemia further changes platelet function by impairing calcium homeostasis and thereby alters aspects of platelet activation and aggregation, including platelet conformation and release of mediators. In diabetes, plasma coagulation factors (e.g., factor VII and thrombin) and lesion-based coagulants (e.g., tissue factor) are increased, and endogenous anticoagulants (e.g., thrombomodulin and protein C) are decreased. In 
addition, the production of plasminogen activator inhibitor-1, a fibrinolysis inhibitor, is increased. Thus, a propensity for platelet activation and aggregation, coupled with a tendency for coagulation, is relevant to a risk of thrombosis complicating plaque rupture (Fig. 7) ${ }^{(13)}$.

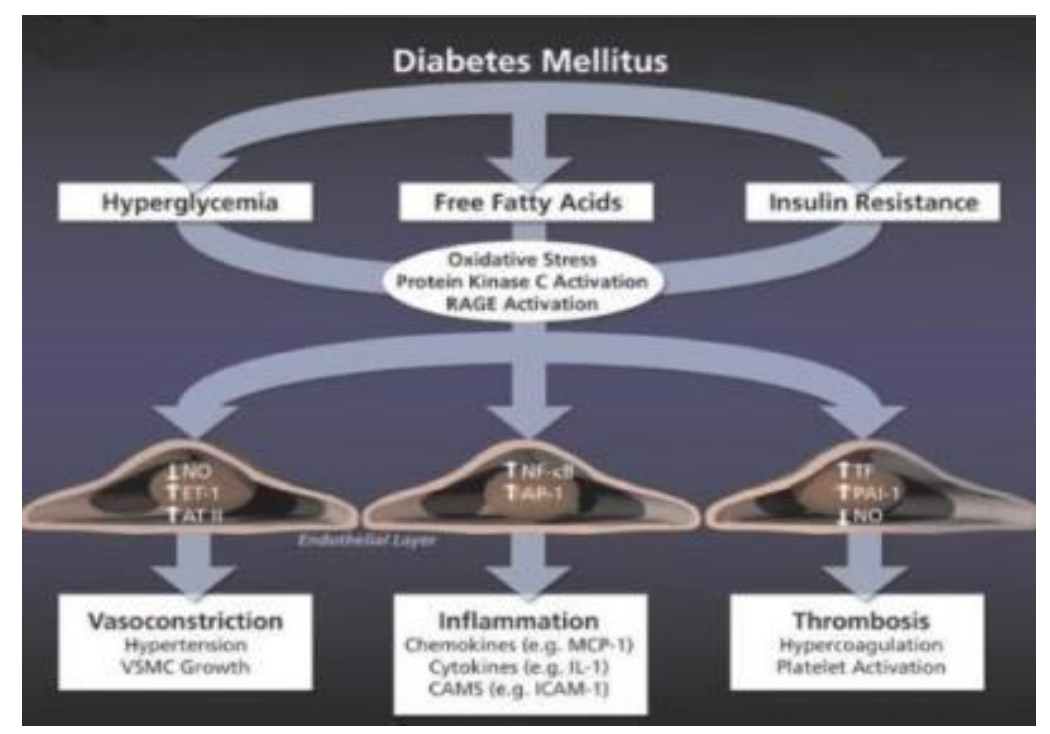

Fig. 7: the metabolic abnormalities that characterize diabetes ${ }^{(11)}$

\section{Classification of Foot Ulcers:}

Many different classification systems were reported in the literature. The one developed by Wagner for grading diabetic foot ulcers has been widely used and accepted. The University of Texas group has developed an alternative classification system that, in addition to ulcer depth (as in the Wagner system), takes into account the presence or absence of infection and ischemia ${ }^{(14)}$.

\section{Wagner Diabetic Foot Ulcer Classification System}

Table (1): Assesses ulcer depth and the presence of osteomyelitis or gangrene) ${ }^{(\mathbf{1 4})}$.

\begin{tabular}{|c|l|}
\hline Grade & \multicolumn{1}{|c|}{ Description } \\
\hline 0 & $\begin{array}{l}\text { No ulcer, but high-risk foot (e.g., deformity, callus, } \\
\text { insensitivity) (Charcot's foot) }\end{array}$ \\
\hline 1 & Superficial full-thickness ulcer \\
\hline 2 & Deeper ulcer, penetrating tendons, no bone involvement \\
\hline 3 & Deeper ulcer with bone involvement, osteitis \\
\hline 4 & Partial gangrene (e.g., toes, forefoot) \\
\hline 5 & Gangrene of whole foot \\
\hline
\end{tabular}

\section{University of Texas Wound Classification System:}

Table (2): Assesses ulcer depth, the presence of wound infection and the presence of clinical signs of lowerextremity ischemia ${ }^{(14) .}$

\begin{tabular}{|l|c|c|c|c|}
\hline Stage & Grade 0 & Grade 1 & Grade 2 & Grade 3 \\
\hline A & $\begin{array}{c}\text { Pre-ulcer or post- } \\
\text { ulcer lesion; no skin } \\
\text { break }\end{array}$ & Superficial ulcer & $\begin{array}{c}\text { Deep ulcer to } \\
\text { tendon or } \\
\text { capsule }\end{array}$ & $\begin{array}{c}\text { Wound } \\
\text { penetrating bone or } \\
\text { joint }\end{array}$ \\
\hline B & + Infection & + Infection & + Infection & +Infection \\
\hline C & + Ischemia & + Ischemia & + Ischemia & +Ischemia \\
\hline
\end{tabular}




\begin{tabular}{|l|c|c|c|c|}
\hline $\mathrm{D}$ & $\begin{array}{c}\text { + Infection and } \\
\text { ischemia }\end{array}$ & $\begin{array}{c}\text { + Infection and } \\
\text { ischemia }\end{array}$ & $\begin{array}{c}\text { +Infection and } \\
\text { ischemia }\end{array}$ & $\begin{array}{c}\text { +Infection and } \\
\text { ischemia }\end{array}$ \\
\hline
\end{tabular}

Techniques of different modalities

Musculoskeletal imaging techniques:

X-RAY of the foot:

Routine Views:

1)Antero-posterior

2)Internal Oblique

3)Lateral

4)Additional film of toe sometimes needed ${ }^{(\mathbf{1 5})}$.
1) Antero-posterior (Fig.8)

1. $\quad 10 \times 12$ detail film divided lengthwise.

2. Table top

3. Patient's foot plantar surface (sole) of foot resting on film. Angle 10 toward the heel.

4. Central Ray: perpendicular to metatarsals (15).
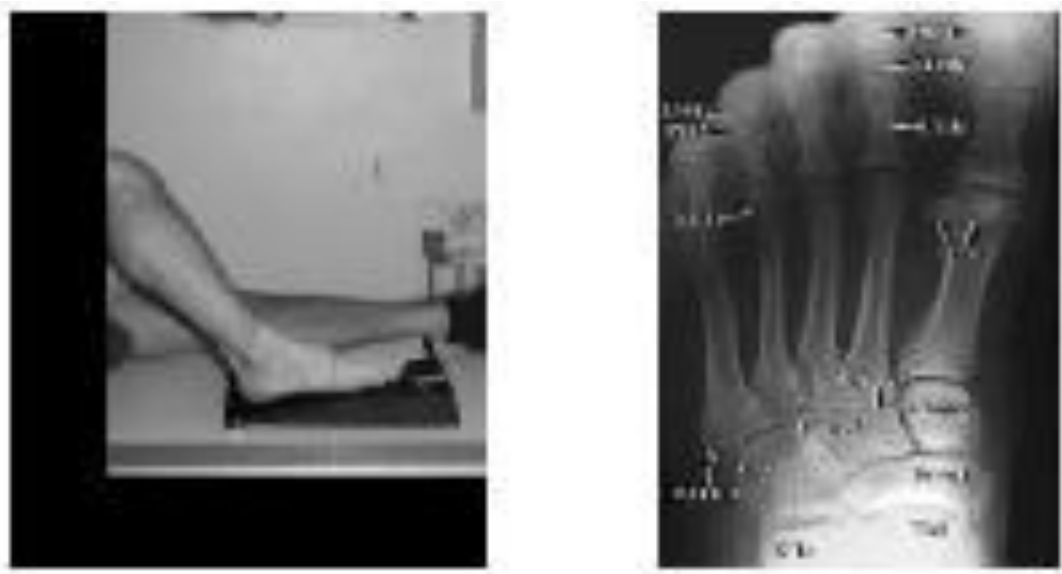

Fig. 8: anterior posterior view positioning and image ${ }^{(\mathbf{1 5})}$

2) Internal Oblique (Fig.9):

1. $10 \times 12$ detail film divided lengthwise.

2. Table top

3. Rotate foot medially 45.

4. Central Ray: centered to base of the $3^{\text {rd }}$ metatarsals ${ }^{(\mathbf{1 5})}$
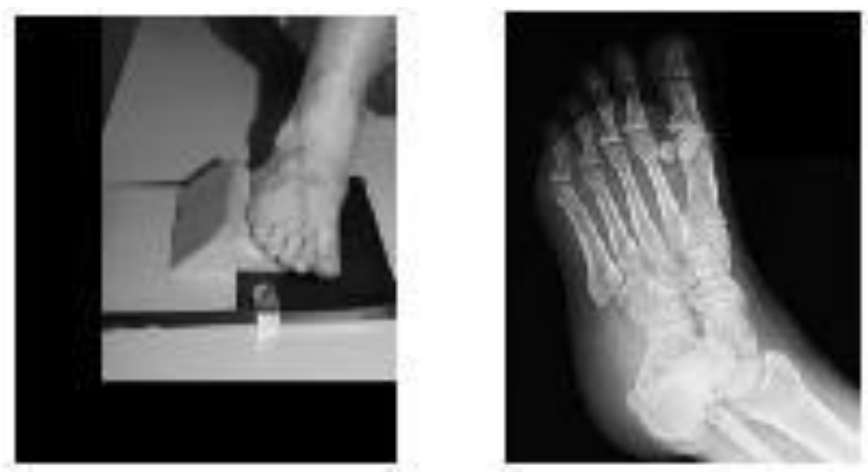

Fig. 9: oblique lateral view positioning and image ${ }^{(15)}$

3) Lateral (Fig.29):

1. $10 \times 12$ detail films or $8 \times 10$ detail films

2. Table top

3. Turn toward lateral side (true lateral). Support under knee.

4. Central Ray: centered to base of 3rd metatarsal ${ }^{(\mathbf{1 5})}$. 

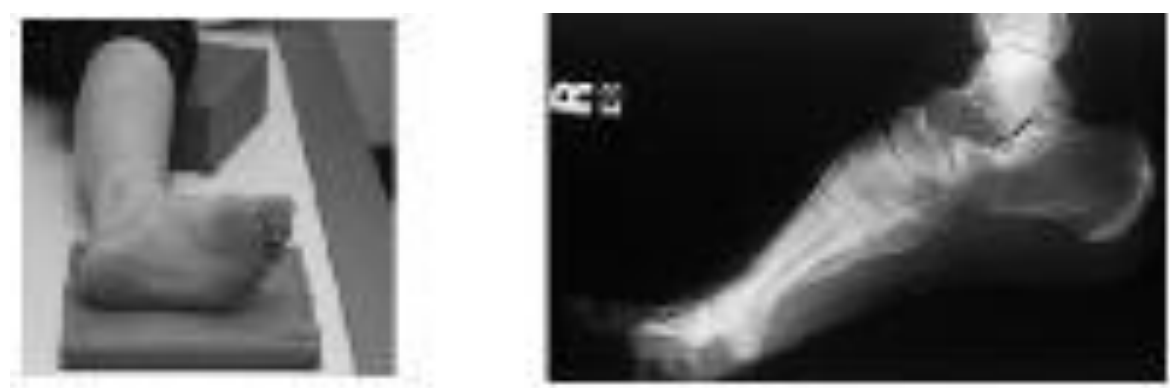

Fig 10: lateral view positioning and image ${ }^{(15)}$

\section{Computerized tomography ${ }^{(16)}$}

\section{Patient Position:}

- $\quad$ Patient supine.

- Center in scanner both feet and foot of interest.

- $\quad$ Use foot holder if available.

- If imaging both feet, bring them together.

- Toes pointing straight up.

- Foot inverted slightly.

\section{Parameters:}

- Gantry Tilt=0

- $\quad$ Field of view: small

- $120 \mathrm{KV}$ (Kilo Volt), 200 MA (MilleAmpere).

- $\quad$ Slice thickness $0.6 \mathrm{~mm}$ interval ${ }^{(\mathbf{1 6})}$.

Axial CT is a Two Dimensional (2D) crosssectional imaging method. In regions of complex anatomy such as the ankle and hind foot, axial CT

(a)

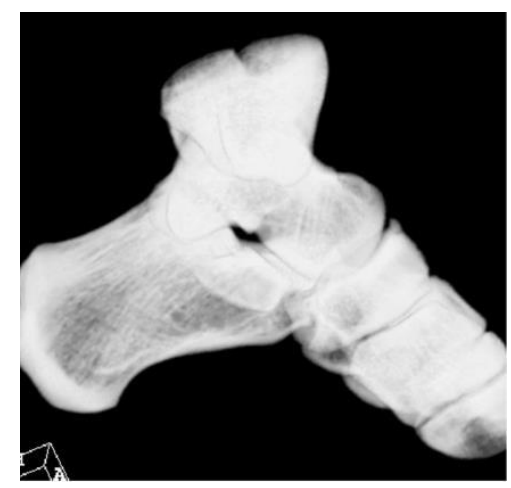

Fig.11: VR imaging of the foot and ankle (a) VR image of the bones depicts the osseous structures of the foot and ankle. (b) VR image of the bones and tendons demonstrates the relationship between the two types of tissue ${ }^{(\mathbf{1 7})}$

\section{Technical Limitations of 3D Rendering:}

Fine detail can be lost with 3D rendering. With SSD and MIP, large portions of the original data set are discarded. With VR, most of the voxel information present in the original data set is scans can be challenging to interpret. For example, the precise location of the articular facets of the subtalar joints may be difficult to appreciate on routine axial scans. Two-Dimensional MultiPlanar Reformatted (2DMPR) images are the basic images used for diagnosis. MPR images are especially useful for identifying small fractures. Volume Rendering (VR) is useful for demonstrating the relationships between ankle tendons and the underlying osseous structures and Shaded Surface Display (SSD) is useful when fractures extend to the articular cortex and a disarticulated view is desired. Three-dimensional images are helpful in patients with congenital deformities, arthritis and trauma ${ }^{(16)}$. The major advantage of VR (Fig.11) is its capacity to display multiple tissues and show their relationships to one another. VR programs retain all of the information within the CT data set and display one or more tissues depending on the chosen protocol. This technique is helpful displaying the relationships of bone to soft tissue ${ }^{(\mathbf{1 0})}$.

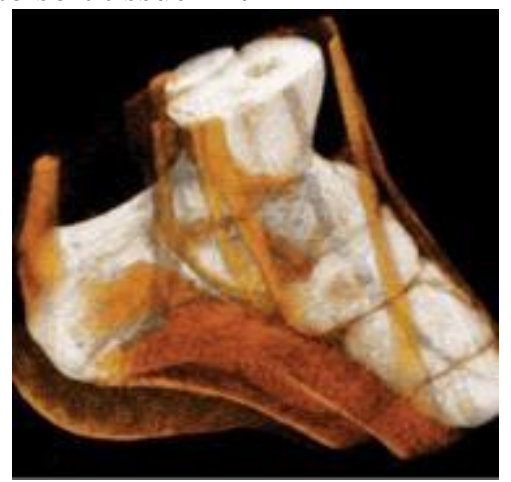

(b)

retained, so that detailed structures can sometimes be identified. Unfortunately, unless image editing is performed, overlapping structures can obscure some of the detail in the region of interest, underscoring the need to review VR images from multiple perspectives. Consequently, primary diagnosis of bone abnormalities (eg, fractures) is best accomplished with the source CT scans or MPR images .Three-dimensional images are useful in providing a global depiction of the bones 
and other tissues when complex fractures or anatomic relationships are present (Fig. 12) ${ }^{(16)}$.

(a)

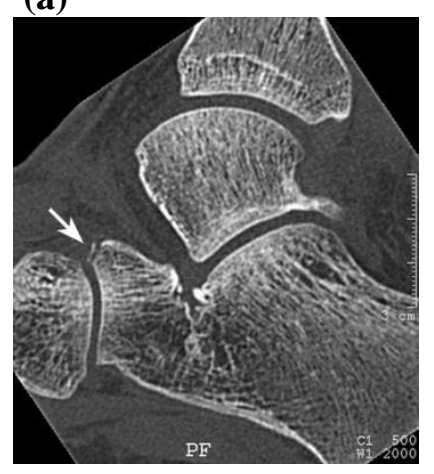

(b)

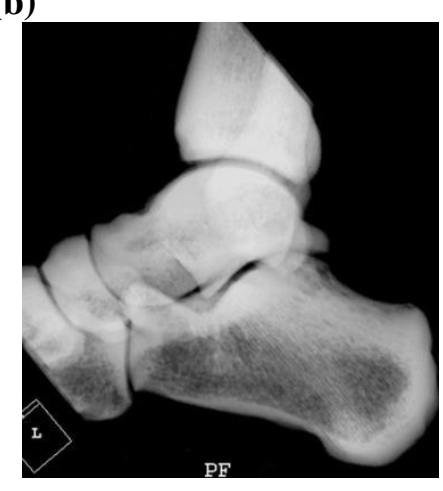

(c)

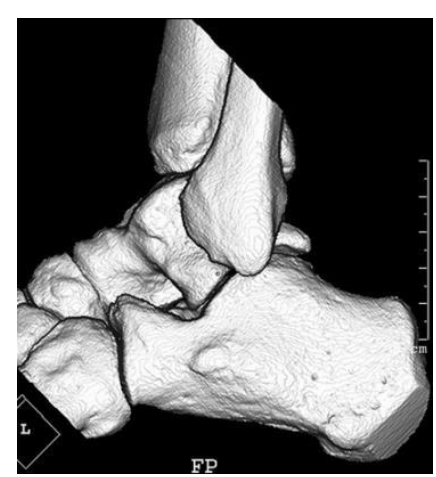

Fig. 12: limitations of 3D rendering technique. (a) Sagittal MPR image obtained in a 23-year-old man who sustained an acute twisting injury and presented with pain and swelling of the left ankle shows a small fracture of the anterior process of the calcaneus (arrow). (b, c) Neither a VR image of the bones (b) nor an SSD image (c) demonstrates the anterior process fracture ${ }^{(\mathbf{1 7})}$

\section{CT Technique:}

Single-phase contrast material-enhanced helical CT is performed following injection of $125 \mathrm{~mL}$ of a low-osmolarity iodinated contrast medium at a rate of $4 \mathrm{~mL} / \mathrm{sec}$ by using a power injector. The study is performed with the patient breathing quietly. Typical scanning parameters would be a collimator width of $5.0 \mathrm{~mm}$, pitch of 1.5 , gantry rotation time of 0.8 second, and field of view of 50 $\mathrm{cm}$. The helical data are retrospectively reconstructed at 2-4 mm intervals ${ }^{(\mathbf{1 6})}$.

\section{PET Technique:}

The combination of CT or MR imaging with PET imaging synergistically maximizes the diagnostic potential of a combined structural-functional imaging approach for the detection, characterization, and monitoring of myriad musculoskeletal disorders ${ }^{(13)}$.

The PET scanner is located behind the CT scanner and housed in the same extended-length gantry. PET is performed following the CT study without moving the patient. Approximately six to seven bed positions are planned in the three-dimensional acquisition mode for scanning the entire patient with 5-7 minute acquisition at each bed position. The maximum length of the patient that can be scanned with the current PET-CT scanner is 145 cm compared with $100 \mathrm{~cm}$ on the prototype. Each bed position (in the cranio-caudal direction) is 15.5 $\mathrm{cm}$ long, and the table moves $11.5 \mathrm{~cm}$ following acquisition of data at each bed position - there is approximately a $4 \mathrm{~cm}$ overlap between table stations. The field of view is $58.5 \mathrm{~cm}$, the spatial resolution is approximately $5 \mathrm{~mm}$, and the sections are post-processed to a thickness of 2-4 $\mathrm{mm}$ for fusion with CT images ${ }^{(16)}$.

\section{Imaging the Foot in the Diabetic Patients:}

Amputation and pedal bypass graft surgery may be the only treatment option for limb salvage in diabetic patients at risk of limb loss. Therefore, careful evaluation of proximal vessels and potential touchdown sites is essential for surgical planning. For the latter, high spatial resolution is particularly important because of the potentially small caliber of the touchdown site. At the same time, this clinical scenario often requires high temporal resolution because of venous contamination in critical limb ischemia patients. This is further complicated in the setting of cellulitis or ulcerations, where the arterial flow is faster that is, there is more early venous enhancement. Therefore a dedicated high spatialresolution calf and foot MRA, preferably timeresolved MRA, in these patients with the goal of showing early or arterial phase pedal soft tissue enhancement that commonly occurs at weightbearing sites of the foot, even when a superficial abnormality is not present ${ }^{(16)}$. Fresh blood imaging is an ECG-gated 3D fast spin-echo strategy that acquires images over one acquisition by triggering in both systole and diastole, thus allowing separation of arteries from veins. Diastolic triggering is applied to visualize fresh blood that enters the veins and arteries, where the flow is relatively slow in comparison with systole and during which only venous flow is typically depicted. In large vessels such as the aorta, the difference in flow rates between systole and diastole is large enough to perform subtraction to 
separate arteries from veins and to depict both vascular systems. In the slower-flow peripheral vessels, additional flow-spoiling pulses are required to yield bright blood during diastole and black blood during systole, MIP of the subtracted (diastolesystole) images eliminates venous and background signals for interpretation. Although this and future methods hold great promise, clinical trials are necessary to determine whether the technique is suitable to routinely separate slow flow in a population of patients with peripheral arterial disease ${ }^{(\mathbf{1 6})}$.

\section{Radiological Manifestations of the Diabetic Foot}

Advanced imaging of the foot has improved the ability to evaluate the possibility of osteomyelitis. The value of a diagnostic modality depends not only on what needs to be known but also on when it is needed to be known and the level of diagnostic certainty required. Such considerations have become increasingly important in the current climate of concern over healthcare costs and the appropriate use of technology ${ }^{(9)}$. The infected or potentially infected diabetic foot can be a difficult management problem. Peripheral neuropathy can diminish symptoms and thus mask the severity of the disease. Patients often present with visible evidence of soft tissue infection either ulcers or cellulites. The presence of osteomyelitis complicates treatment and may decrease the probability of a successful outcome. Helpful clinical features that increase the probability of osteomyelitis are an ulcer with a cross-sectional area greater than $2 \mathrm{~cm}^{2}$, extending down to bone and an ESR $>70 \mathrm{~mm} /$ hour $^{(\mathbf{9})}$. Diagnostic imaging would add value if it provide increased assurance that osteomyelitis was or was not present but only if it resulted in action that otherwise would not have been taken. The diagnostic value of various imaging studies in diabetic foot infections has been reviewed extensively ${ }^{\left({ }^{(18)}\right.}$. CT should be used as a complementary technique to visualize bony destruction or bony sequestration. Reconstructed 3-D images can aid in planning surgery ${ }^{(\mathbf{1 8})}$. MRI is the best imaging technique because it is highly sensitive in detecting both soft-tissue lesions (skin ulcers and tracts, cellulites and abscesses) and bone lesions (hypo-intense replacement of bone marrow on T1-weighted images). MRI can also stage diabetic foot lesions accurately. Postcontrast images can provide additional information (rim abscess enhancement and diffuse cellulites enhancement), but coexistent renal failure in those patients must be kept in mind (18).

\section{Imaging manifestations of Charcot's neuro arthropathy:}

It is a sudden softening of the bones in the foot that can occur in patients with neuropathy. The bones are weak enough to fracture and with continued walking the foot eventually changes shape. As the disorder progresses, the arch collapses and the foot takes on a convex shape, giving it a rocker-bottom appearance, making it very difficult to walk. Charcot foot is a very serious condition that can lead to severe deformity, disability and even amputation ${ }^{(9)}$. Numerous classification systems based on clinical, radiographic and anatomic pathology describe Charcot arthropathy. Anatomic classification systems are the most commonly used and have the added benefit of predicting outcome and prognosis ${ }^{(9)}$.

\section{Radiological manifestations of diabetic foot infections: \\ Plain film Radiography}

Radiographs are always performed first because they may provide the diagnosis by showing trabecular or cortical lysis. They also give an overview of the anatomy and any underlying complicating structural changes, such as that occurring in diabetic arthropathy; however in many cases it is not helpful except in identifying edema and gas in the soft tissues in gas-forming infections (Fig. 13) ${ }^{(19)}$.

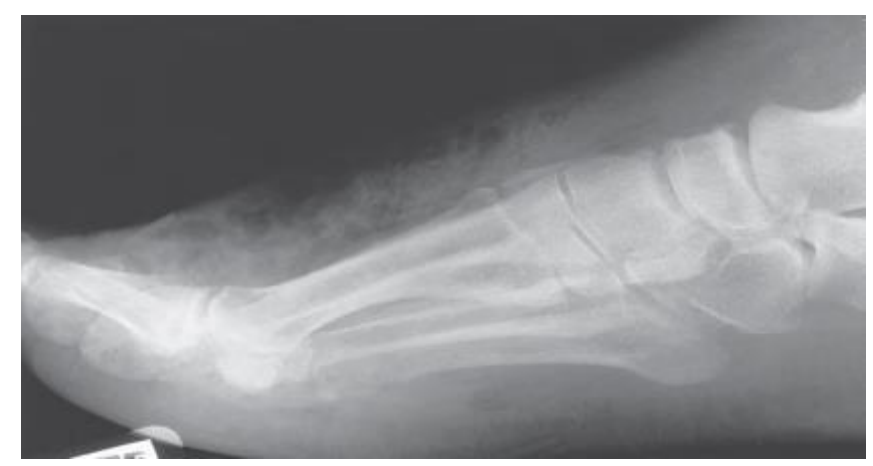

Fig.13: diffuse soft tissue swelling and streaky soft tissue emphysema (arrows) from a gasforming organism ${ }^{(1)}$

\section{The ultrasound}

The initial appearance may be generalized swelling and increased echogenicity of the skin and subcutaneous tissues (Fig.14). As cellulitis progresses and the amount of subcutaneous fluid increases, hyper-echoic fat lobules become separated by hypo-echoic fluid-filled areas. This later stage of cellulitis is most typical and has been described as having a cobblestone appearance 
(Fig.15) (not specific for cellulitis but suggests generalized edema). Color and power Doppler are advanced ultrasound modalities that can help determine whether the inflammation characteristic of cellulitis is present ${ }^{(20)}$.

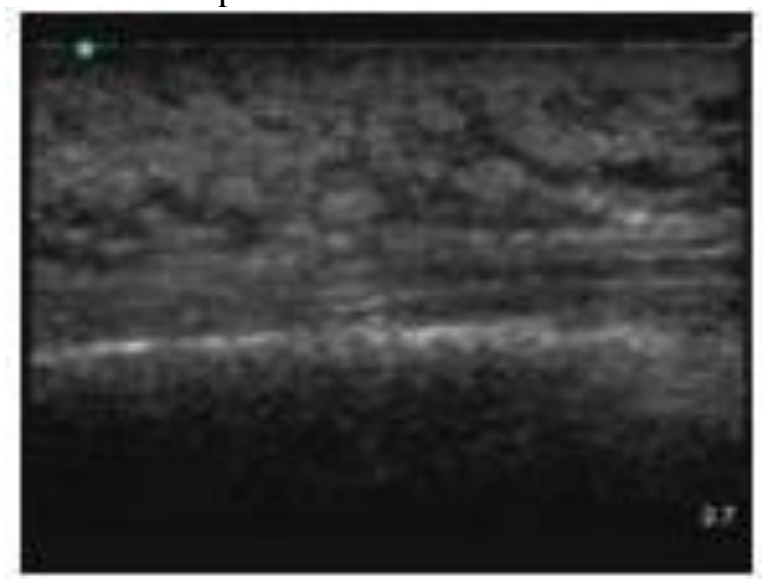

Fig. 14: ultrasound examination showing early cellulitis ${ }^{(21)}$

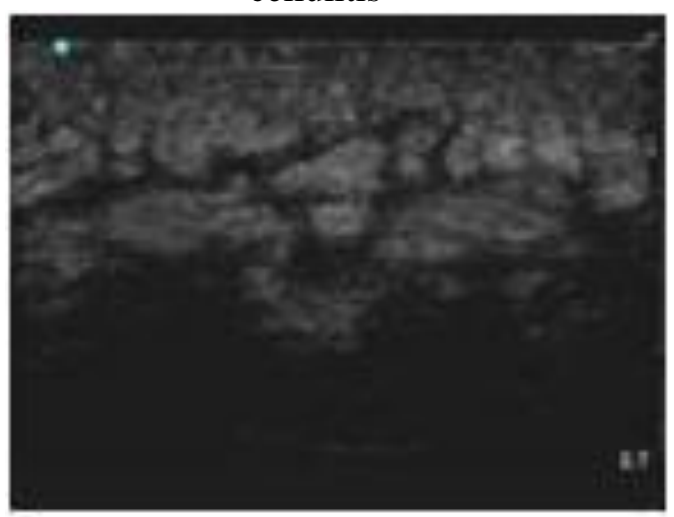

Fig. 15: ultrasound examination Cobblestone appearance of advanced cellulitis ${ }^{(21)}$

The main aim of vascular imaging in the diabetic patients ${ }^{(22)}$
1) Screening:
"Does

Peripheral Arterial Disease Exist?

2) Assessment of Location and Severity.

3) Prognosis and Medical Therapeutic Implications.

4) Perioperative Evaluation.

5) Selection of the Arterial Reconstructive Procedure.

6) Postoperative Follow-up.

7) Healing Response.

\section{Ultrasonography}

Arterial ultrasound examination has become very popular. It is a simple and valid method of low cost to determine the site and degree of obstructive lesions and the patency of a vessel after revascularization. Ultra sonographic equipment used for these tasks includes B -mode imaging, pulse-wave Doppler, continuous wave Doppler and color Doppler display ${ }^{(22)}$. The site of an arterial stenosis can be identified by serial placements of the Doppler probe along the extremities, the exact site of arterial disease being located by the use of duplex scanning. Duplex scanners use the combination of real-time B-mode ultrasound imaging of the arterial wall together with the pulsed Doppler and examine flow patterns in a defined area within the artery lumen. The pulsed Doppler technique performs a spectral analysis of the pulse wave, which delineates the complete spectrum of frequencies (that is blood flow velocities) found in the arterial waveform during a single cardiac cycle. Tissues are displayed in tints of gray scale (duplex) on the screen ${ }^{(22)}$.

\section{Reasonable indications for stent placement:}

- $\quad$ Severe residual dissection (flow limiting) after failure of prolonged low pressure inflation.

- Recoil.

- Residual thrombus not removable by aspiration.

Self-expanding stents are better for long lesions and dissections. Balloon expandable stents should be used for shorter lesions ${ }^{(22)}$.

\section{Multi-detector CT angiography:}

In patients with chronic limb-threatening ischemia, the principal goal of treatment is prevention of tissue loss and need for amputation, assessment and promotion of blood flow through the calf arteries. An accurate roadmap to lesions amenable to percutaneous trans-luminal angioplasty or other endovascular techniques and delineation of patent, acceptable target vessels for distal bypass are the challenges of vessel analysis in this advanced disease group. In this respect, "isotropic" image data sets $(<1 \mathrm{~mm})$ and optimum contrast-medium delivery especially with the 64-row MDCT scanners, may provide improved visualization of small crural and pedal vessels in patients with chronic limb-threatening ischemia ${ }^{(22)}$.

Magnetic Resonance Angiography:

MRA has become the preferred imaging technique for the diagnosis and treatment planning of patients with PAD in many centers. It is useful for treatment planning prior to intervention and in assessing suitability of lesions for endovascular approaches ${ }^{(23)}$.The main advantage of MRA is its ability to provide rapid highresolution threedimensional (3D) imaging of the entire abdomen, pelvis and lower extremities in one setting (Fig.16) (24). 

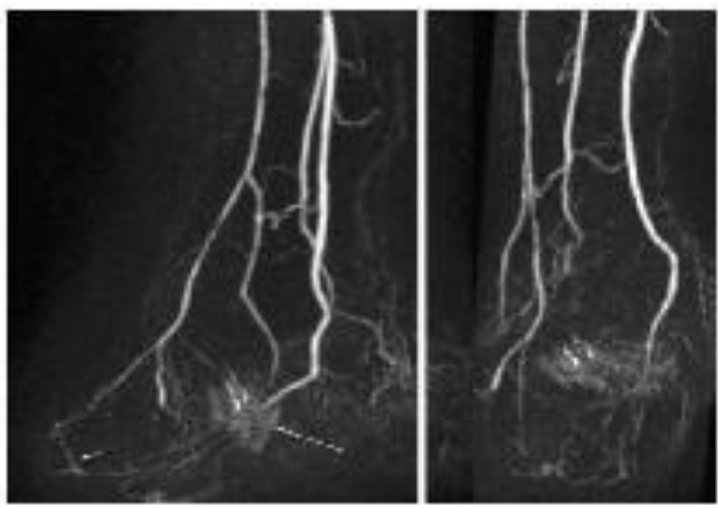

Fig. 16: right foot sagittal (a) and coronal (b) MIP's from a 58 year old male diabetic status post transmetatarsal amputations with amputation site ulcers and osteomyelitis. The pedal inflow is relatively normal (peroneal, anterior and posterior tibial arteries). The dorsalis pedis is somewhat attenuated distally, and there is a severely diseased primary plantar arch (short arrow). The lateral plantar terminates proximally into a region of ulcer blush (long arrows) ${ }^{(24)}$

Furthermore, image volumes can be rotated and assessed in an infinite number of planes. A recent advance in this technique is the development of gadolinium Contrast-Enhanced MRA (CE-MRA) (Fig.17), which has replaced non-contrast MRA for the assessment of peripheral vessels, as it provides rapid imaging with better artifact-free images. CE-MRA has been shown to have better discriminatory power than color-guided duplex ultrasound for the diagnosis of PAD ${ }^{(24)}$.

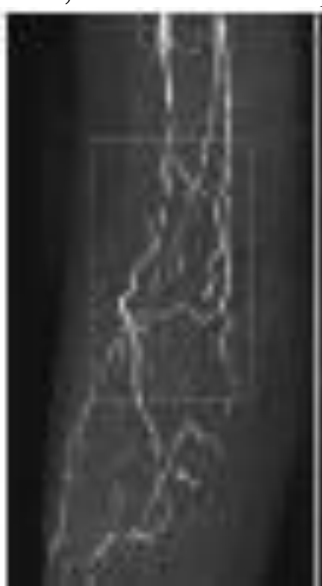

a

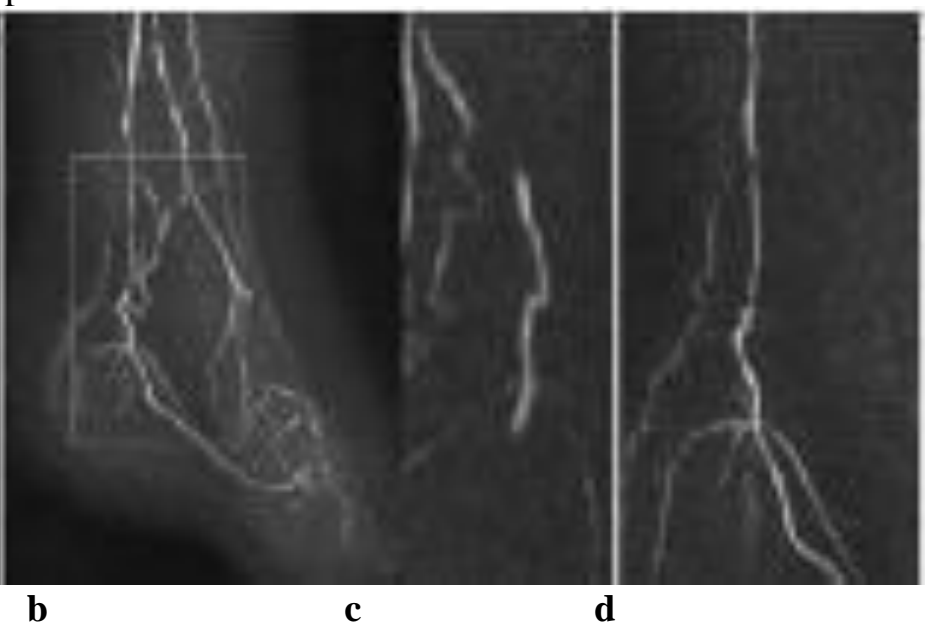

Fig. 17: coronal (a) and sagittal (b) left foot CEMRA MIP's from a 77 year old diabetic male with non-healing foot ulcers. The dorsalis pedis artery is occluded, with a short distal segment reconstituting (arrow), but no patent pedal arch. Diffuse multivessel disease is seen, particularly involving the posterior tibial artery, which is better seen on the coronal (c) and sagittal (d) zoomed sub-volume MIP's (24)

\section{The limitations of CE-MRA are:}

1) The high magnetic field strength, which excludes its use for patients with, e.g. defibrillators, spinal cord stimulators, intracerebral shunts and cochlear implants

2) Cannot be used for patients affected by claustrophobia and patients who are not amenable to sedation.

3) Signal loss in the presence of stents ${ }^{(25)}$.

\section{Conclusion:}

The major complication facing patients with diabetic foot is amputation that is why tight glycemic control is mandatory. In addition to routine screening for early detection of diabetic foot complications can be helpful. With advancement of radiological techniques, the prognosis of diabetic foot has improved.

\section{References:}

1. Paydar KZ, Hansen SL, Charlebois ED et al. (2006): Inappropriate antibiotic use in soft tissue infections. Arch. Surg., 141:850856.

2. Dutta $\mathbf{P}$, Bhansali A, Singh $P$ et al. (2004): Charcot's foot: advanced manifestation of diabetic neuropathy. Postgrad. Med. J., 80:433-434.

3. Butalia S, Palda VA, Sargeant RJ et al. (2008): Does this patient with diabetes have osteomyelitis of the lower extremity. JAMA., 299(7): 806-813. 
4. Sutton D and Roger H S (2003): Arteriography and interventional angiography. In: Textbook of Radiology and Imaging. Elsevier Science Ltd. $7^{\text {th }}$ ed. London. pp: 215263.

5. O'Neil J and Glickman A (2008): Ankle and foot, musculoskeletal ultrasound. $1^{\text {st }}$ ed. Springer Science Brownes Media LLC. London. pp: 243-256.

6. Hope CM, Harley BK, Randy C et al. (2010): FDG-PET A better imaging option for diabetic osteomyelitis. International Journal of Radiology, 23(6): 18-22.

7. Hensen J (2010): Netter's clinical anatomy. Saunders Elsevier. $2^{\text {nd }}$ ed. London. pp: 203-208.

8. Gray $H$ and Lewis $W$ (2008): Anatomy of the Human Body, Syndesmology. Lea and Febiger, $40^{\text {th }}$ ed. Philadelphia. pp: 312-325.

9. Ryan DH and Cohen HJ (2005): Bone marrow examination. In: HematologyBasic Principles and Practice. $4^{\text {th }}$ ed. Elsevier Churchill Livingstone. Philadelphia. pp: 2556-2672.

10. Beltran J (2010): Clinical Magnetic Resonance Imaging. Ankle and foot. $3^{\text {rd }}$ ed. Elsevier.London. pp: 260-283.

11. Tentolouris N, Katsilambros N, Dounis E et al. (2010): Atlas of the Diabetic Foot. John Wiley and Sons. $2^{\text {nd }}$ ed. Ltd. Publication. London. pp: 1-5.

12. Mcphee $\mathrm{S}$, Hammer $\mathrm{G}$, Holder $\mathrm{J}$ et al. (2010): Pathophysiology of disease: an introduction to clinical medicine. In: Disorders of Endocrine Pancreas. $6^{\text {th }}$ ed. The McGrawHill Companies. London. pp: 218-255.

13. Hofmann M, Pichler B, Scholkopf B et al. (2009): Towards quantitative PET/MRI: a review of MR-based attenuation correction techniques. Eur. J. Nucl. Med. Mol. Imaging, 36(1): 93-104.

14. Brownlee $M$, Aiello $L$, Cooper $M$ et al. (2011): Complications of diabetes mellitus.In:Williams Textbook of Endocrinology. $2^{\text {th }}$ ed. Elsevier.London. pp: 1532-1533.
15. Russell JA, Shave RM, Kruse DW et al. (2011): Ankle and foot contributions to extreme plantar- and dorsiflexion in female ballet dancers. Foot Ankle Int., 32(2):183-188.

16. Kapoor V, McCook B and Torok $F$ (2004): An introduction to PET-CT imaging. Radiographics, 24: 523-543.

17. Pelc JS and Beaulieu CF (2001): Volume rendering of tendon-bone relationships using unenhanced CT. AJR. Am. J. Roentgenol., 176:973-977.

18. Berscquet $T$, Tomas $X$ and Garcia $A$ (2011): Imaging of the Ankle and Foot. Ankle and Foot. $3^{\text {rd }}$ ed. Lipincott William and Wilkins. London.pp: 218-220.

19. Miller T and Scgweitzer M (2016): Workshops of musculoskeletal disease infections. Diabetic foot. In: Musculoskeletal Disease. $2^{\text {nd }}$ ed. Springer-Verlag Italia. pp: 149-150.

20. Chau CL and Griffith JF. (2005): Musculoskeletal infections: ultrasound appearances. Clin. Radiol., 60(2):149-159.

21. Nazarian LN. (2008): The top 10 reasons musculoskeletal sonography is an important complementary or alternative technique to MRI. American Journal of Roentgenol., 190(6):1621-1626.

22. Frykberg R, Zgonis T, Armstrong D et al. (2006): Diabetic foot disorders, a clinical practice guideline. The Journal of Foot and Ankle Surgery, 45(5): 7-9.

23. Goyen M (2008): Gadofosveset enhanced magnetic resonance angiography. Vascular Health Risk Management, 4:1-9.

24. Hadizadeh DR, Gieseke J, Lohmaier SH et al. (2008): Peripheral MR angiography with blood pool contrast agent: Prospective intraindividual comparative study of high-spatialresolution steadystate MR angiography versus standardresolution firstpass MR angiography and DSA. Radiology, 249:701-711.

25. Norgren L, Hiatt WR, Dormandy JA et al. (2007): Inter-society consensus for the management of peripheral arterial disease (TASC II). J. Vasc. Surg., 45: 5-67. 